\title{
GROUPS WITH CYCLIC DERIVED FACTOR-GROUP
}

\author{
HERMANN HEINEKEN, JOHN C. LENNOX, \\ A. G. R. STEWART AND JAMES WIEGOLD
}

(Communicated by Bhama Srinivasan)

\begin{abstract}
In [1] it is proved that a $k$-generator metabelian group with $G / G^{\prime}$ cyclic can be generated by $k$ conjugates of a single element, and the problem was left over as to whether this is true for soluble groups in general. This paper provides counterexamples of Fitting length 3, as well as proving that the result of [1] is true whenever all subgroups of $G^{\prime}$ are subnormal.
\end{abstract}

We begin with the results mentioned in the abstract.

Theorem. Let $G$ be a group with $G / G^{\prime}$ cyclic and $d(G)=k$, and suppose that every subgroup of $G^{\prime}$ is subnormal. Then $G$ is generated by $k$ conjugates of every element generating $G$ modulo $G^{\prime}$.

Proof. Set $G=\left\langle a, G^{\prime}\right\rangle$. Tietze transformations bring $G$ to the form $G=$ $\left\langle a, x_{1}, \ldots, x_{k-1}\right\rangle$ with $x_{i}$ in $G^{\prime}$ for each $i$. Consider the subgroup $K=$ $\left\langle a,\left[a, x_{1}\right], \ldots,\left[a, x_{k-1}\right]\right\rangle$. The metabelian argument in [1] shows that $G=$ $K G^{\prime \prime}$, so that $G^{\prime}=K^{\prime} G^{\prime \prime}$. But $K^{\prime}$ is subnormal in $G^{\prime}$, and thus $G^{\prime}=K^{\prime}$ by Lemma 5 of [2]. Then $G=K$ and $G=\left\langle a, a^{x_{1}}, \ldots, a^{x_{k-1}}\right\rangle$, as required. Note that the theorem holds when $G^{\prime}$ is nilpotent, and thus when $G$ is supersoluble.

Next, we construct our counterexamples. Let $p$ and $r$ be primes, not necessarily different; and let $q$ be a prime dividing $r^{p}-1$ but not $r-1$. In this case $q-1$ is a multiple of $p$, so that there is a non-abelian group $H$ of order $p q$. Let $A$ be a faithful irreducible $H$-module over the field $F$ of order $r$. The automorphism group of the $H$-module $A$ is isomorphic to the multiplicative group of $F$.

Let $B$ be the direct product of $p$ copies of $A$ and $D$ be the direct product of $p-1$ copies of $A$. We shall need the two semidirect products $G=B H$ and $K=D H ; G$ is the required counterexample.

Lemma 1. $d(G)=1$.

Received by the editors August 14, 1987 and, in revised form, May 15, 1989.

1980 Mathematics Subject Classification (1985 Revision). Primary 20F05, 20E15, 20 D10. 
Proof. Let $v$ be a generator of the $H$-module $B$, and set $H=\langle x, y\rangle$, with $x$ of order $p, y$ of order $q$, and $y^{x}=y^{m}$. Then $\langle x v, y\rangle=\left\langle x v, y, v^{-1} x^{-1} y x\right\rangle=$ $\left\langle x v, y, v^{-1} y^{m} v\right\rangle=\left\langle x v, y,\left[y^{m}, v\right]\right\rangle=\langle x v, y, v\rangle=\langle x, y, v\rangle=G$; the fourth equality comes from the fact that $y$ operates fixed-point freely on $B$.

Lemma 2. $K$ cannot be generated by two elements of order $p$.

Proof. Since $K / D$ is non-cyclic, every pair of generators for $K$ consists of elements outside $D$. It suffices to look at groups $W=\langle x, x y d\rangle$ with $x$ and $y$ as in Lemma 1 , and $d$ in $D$. Since $(x y d)^{p}=1$ and $x y$ operates fixedpoint freely on $D$, we find that $d=[x y, w]$ for some $w$ in $D$, so that $W=\left\langle x, w^{-1} x y w\right\rangle$.

Recall that $D$ is a direct product of $p-1$ copies of the faithful irreducible $H$-module $A$, and that every isomorphism will map the intersection of one of the direct factors with $C_{D}(x)$ onto the corresponding intersection. The same goes for $C_{D}(x y)$, and there is a complement $C$ of $C_{D}(x) C_{D}(x y)$ in $D$ with the same property. Thus $w$ can be written uniquely as $w=a b c$, with $c$ in $C, a$ in $C_{D}(x)$ and $b$ in $C_{D}(x y)$. Now

$$
a W a^{-1}=\left\langle a x a^{-1}, c^{-1} b^{-1} x y b c\right\rangle=\left\langle x, c^{-1} x y c\right\rangle \subseteq\langle x, y, c\rangle .
$$

Since the intersection of $C$ with each of the direct factors of $D$ has order $r^{p-2}, c$ cannot generate $D$ as $H$-module. Thus $a W a^{-1}$ is not $K$, nor is $W$, and this completes the proof.

Proposition 3. $G$ cannot be generated by two conjugate elements.

Proof. Since $K$ is a quotient of $G$, we see immediately that $G$ cannot be generated by two elements of order $p$. If $G=\left\langle z, h^{-1} z h\right\rangle$, then $z$ must be of order $p r$. But then $z^{p}=a$ would centralize $z$, and $G /\langle a\rangle^{G}=K$ would be generated by two elements of order $p$. This contradicts Lemma 2, and the argument is complete.

\section{ACKNOWLEDGMENT}

The authors express their sincere thanks to the referee. Our original example was the case $p=r=2$ and $q=3$, so that his/her remarks have resulted in a very much improved article.

\section{REFERENCES}

1. J. L. Brenner, R. M. Guralnick and James Wiegold, Two-generator groups III, Contemp. Math. 33 (1984), 82-89.

2. J. E. Roseblade, The permutability of orthogonal subnormal subgroups, Math. Z. 90 (1965), $365-372$.

Mathematics Institute, 87 Würzburg, Universitat Würzburg, Federal Republic of GeRMANY

(Address of J. C. Lennox and J. Wiegold) University of Wales, College of Cardiff, CARDIFF CF1 1LX, UNITED KINGDOM

UNiversity OF ZimbabWe, ZimbabWe 